\title{
Global fluctuations in decaying Burgers turbulence
}

\author{
Alain NOULLEZ ${ }^{1}$ and Jean-François PINTON ${ }^{2}$ \\ 1 Laboratoire Cassini CNRS UMR 6529, Observatoire de la Côte d'Azur, B.P. 4229, F-06304 Nice, France \\ 2 Laboratoire de Physique \& CNRS UMR 5672, Ecole Normale Supérieure, 46 Allée d'Italie, F-69007 Lyon, France
}

the date of receipt and acceptance should be inserted later

\begin{abstract}
We consider the statistics of the total energy and the global dissipation in numerical simulations of decaying Burgers turbulence. We observe that at large times these global quantities develop strongly non-Gaussian statistics with asymmetric probability density functions characterized by an exponential tail for values above the mean. Although $\chi^{2}$ distributions would be expected in the description of such definite positive quantities as the energy or the dissipation, we observe that the generalized Gumbel $G_{a}$ functions provide a better functional form. These distributions have an exponential tail which correctly represents the large fluctuations of the energy or dissipation at large times. We find that the value of the control parameter $a(t)$ of the fitting Gumbel distribution $G_{a}$ at time $t$ is set by the ratio of the system size to the velocity correlation length, which thus plays the role of an effective number of degrees of freedom. We show that in this problem, the development of exponential tails for the global variables is linked to extreme statistics properties for the largest local events.
\end{abstract}

PACS. 47.27.Eq Turbulence simulation and modeling - 52.25.Gj Fluctuation phenomena - 02.50.-r Probability theory, stochastic processes, and statistics - 05.70.Ln Nonequilibrium thermodynamics, irreversible processes

\section{Introduction}

In the study of dissipative systems with a large number of degrees of freedom, much work has been devoted to the decription of small scales properties. However in the recent years there has been a growing interest in the behavior of global quantities. More precisely, one has considered the dynamics in time of fields spatially averaged over the system size :

$$
G(t)=\int \mathrm{d}^{3} r g(\mathbf{r}, t) .
$$

In the case of complex dissipative system, a particular attention has been paid to energetical quantities. At any time $t$, the change in the total kinetic energy $E(t)$ results from the balance between injection and dissipation

$$
\frac{\mathrm{d} E(t)}{\mathrm{d} t}=\epsilon_{I}(t)-\epsilon_{D}(t)
$$

where $\epsilon_{I}(t)$ is the power injection if the system is forced and $\epsilon_{D}(t)$ is the dissipation. In turbulent flows or granular flows, the power injection is made at large scale by the motion of boundaries and the dissipation is due to the viscosity at small scale for the fluid or to the inelasticity in the particle shocks for the granular medium.

In any real system, the time average of equation (2) states that injection must balance the dissipation if the system is to achieve a stationary state. This argument, combined with dimensional analysis, usually yields the dependence of the average value of the injection as a function of the system characteristics. However, the quantities in equation (2) fluctuate with time, and the statistical distribution of these fluctuations are of interest. Indeed, given the large scale separation between the system size and the smallest length scale in most systems, it was originally believed that the fluctuations in the global quantities would be Gaussian with a variance that decreases as the inverse of the number of degrees of freedom in the system. It has been shown in a fluid turbulence experiment [18] that this not necessarily the case: confined turbulent flows display non trivial fluctuations in the energy injection. In this case, the probability density function (PDF) $p\left(\epsilon_{I}\right)$ is strongly non-Gaussian with an asymmetric exponential tail for values below the mean. Furthermore, it was observed [22] that the shape of the PDF does not vary significantly with the Reynolds number.

Further understanding of this behavior was made possible by the observation that an identical statistical behavior occurs for the fluctuations of the order parameter in a critical system. Specifically, an identical PDF is observed for the fluctuations in the global magnetization of the 2DXY model at temperatures below the KosterlitzThouless transition [1]. It was then proposed in [4] that the key feature for the development of such non-Gaussian statistics in an equilibrium or a dissipative system is that the correlation length is of the order of the system size. 
Motivated by these observations, global fluctuations have been studied in a large variety of systems and similar asymmetric PDFs with an exponential tail have been reported for self-organized critical sytems [5], Nusselt fluctuations in turbulent convection [2], simulations of granular media [19]. Analytical expressions for the observed asymmetric PDF have been derived for an equilibrium system, the critical 2DXY model [6] and recently for a dissipative system, the Langevin equation [10]. It has also been shown in the case of the magnetic system [23] that the slope of the exponential tail is determined by the ratio of the correlation length to the system size.

Returning to the question of the origin of global energy fluctuations in turbulence, many questions remain open concerning the physical mechanisms responsible for the non-Gaussian statistics. Among these is the role played by the pressure which is a non-local quantity - in an incompressible fluid the pressure is determined via a Poisson equation. Although similar results have been reported in a direct numerical simulation of 3D turbulence in the presence of a large scale shear [24], a comprehensive analysis is lacking in such a full Navier-Stokes problem. A possible alternative is to study a turbulence-like, simpler, model.

We will consider here the one dimensional Burgers equation

$$
\partial_{t} u(x, t)+u(x, t) \partial_{x} u(x, t)=\nu \partial_{x x} u(x, t) .
$$

This was originally introduced by Burgers [7] as a model for Navier-Stokes turbulence. It was later realized that it can be used to describe a large variety of physical problems ranging from acoustics, phase diffusion, aggregation interface growth to the formation of structures in the Universe $[13,15,17,25,26]$. It is the simplest model of nonlinear diffusion corresponding the conservation of Lagrangian velocities, in the limit of vanishing viscosity. In fact Burgers equation shares many features of the Navier-Stokes equation; it has the same type of non-linearity, dissipation and invariance groups. On the other hand it lacks the pressure term. This comes from the fact that it corresponds to a quite different limit of compressible fluid dynamics. The Navier-Stokes equation corresponds to an infinite speed of sound approximation in which the pressure terms keeps the velocity field divergence-free. In Burgers equation the opposite limit is considered: the medium is pressureless, corresponding to a zero sound speed approximation. The velocity is uncoupled from the density which is set by the continuity equation. Shock waves appear in a finite time and control most of the dynamics and statistical properties at large times.

Burgers equation is then an interesting test bench for the behavior of global quantities. The fact that it lacks a pressure term is even an advantage to check for the relevance of the pressure in the dynamics of global variables in Navier-Stokes turbulence. Indeed, the non trivial behavior for the global fluctuations has been attributed to the existence of large scale correlations spanning the system [4]. The pressure, as a nonlocal quantity, would be a possible candidate for maintaining such correlations in a turbulent flow. Burgers equation is also both non-linear and dissipative. This last feature is in contrast with equilibrium systems where the universal behavior of global quantities have been already studied [4-6]. Finally, like the 2DXY model and very much unlike Navier-Stokes equation, the Burgers equation is explicitly integrable. Note that this property does not allow for a complete analytical calculation of its statistical properties but it provides the key for efficient numerical studies.

The paper is organized as follows: we first recall in section 2 the fundamentals of Burgers equation. We then give a phenomenological decription of Burgers turbulence in the confined decaying regime. We introduce the global variables : total kinetic energy $E(t)$ and total dissipation $\epsilon(t)$. In section 3 we present our observations for the statistical properties of $E(t)$ and $\epsilon(t)$. We detail the influence of the growing correlation length as the turbulence decays in time and link our results with the properties of extremal statistics. Finally we give a physical interpretation for the exponential part of the distribution for fluctuations above the mean.

\section{Global quantities in Burgers equation}

In this Section, we shall briefly recall a few standard results about the Burgers equation. For more details, the interested reader is referred to $[8,16,11,13,27,28]$. We also describe the main features of Burgers turbulence and introduce the global quantities under study.

\subsection{Burgers equation}

We consider the initial value problem for the unforced (decaying) one dimensional Burgers equation (3). It is well known that the Burgers equation can be solved explicitly by introducing the velocity potential

$$
u(x, t)=-\partial_{x} \psi(x, t)
$$

and using the Hopf-Cole $[14,9]$ transformation $\psi=2 \nu \log \theta$ that turns Burgers equation

$$
\partial_{t} \psi(x, t)-\frac{1}{2}\left(\partial_{x} \psi(x, t)\right)^{2}=\nu \partial_{x x} \psi(x, t)
$$

into the heat equation

$$
\partial_{t} \theta(x, t)=\nu \partial_{x x} \theta(x, t)
$$

whose solution can be expressed as a convolution integral.

In this paper we are interested in strongly non-linear regimes, where the Reynolds number is very large. We thus consider the solution of Burgers equation in the limit of vanishing viscosities. In that case, we can use a saddlepoint argument to express the solution for the velocity potential at any time $t>0$ as

$$
\psi(x, t)=\max _{x_{0}}\left[\psi\left(x_{0}, 0\right)-\frac{\left(x-x_{0}\right)^{2}}{2 t}\right] .
$$


At small times, the location $x_{0}$ where the maximum is realized will be $x_{0}=x-u\left(x_{0}, 0\right)$, i.e. the initial position of the fluid particle which reaches $x$ at time $t$, with velocity $u\left(x_{0}, 0\right)$. In this view, it is natural to call $x_{0}(x)$ the inverse Lagrangian function. At later times, the maximum will be reached at more than one position of space, showing that initially separated particles have come together. At this time shocks are formed in the velocity profile.

In the limit of vanishing viscosities, Burgers equation is scale invariant, i.e. it is unchanged under the transformation $\left(x \rightarrow \lambda x, t \rightarrow \lambda^{1-h} t\right)$ for any $h$ if the velocity is also rescaled $u \rightarrow \lambda^{h} u$. Thus if the initial conditions are selfsimilar with a given scaling exponent $h$, then the solution at any time $t$ will also be self-similar although the scaling exponent can, and generally will, be different. But the statistical properties of $u$ at time $t_{2}$ and scale $\ell_{2}$ can be derived from those at time $t_{1}$ and scale $\ell_{1}=\ell_{2}\left(t_{1} / t_{2}\right)^{1 / 1-h}$. The distribution of $u$ at time $t_{2}$ is then that of the distribution at time $t_{1}$ of $\left(t_{2} / t_{1}\right)^{h / 1-h} u\left(\ell_{1}, t_{1}\right)$. We thus infer that any characteristic scale of decaying Burgers turbulence varies with time as

$$
\ell(t) \propto t^{1 / 1-h}
$$

if the initial conditions have a scaling exponent $h$.

We are interested in random initial conditions. A convenient choice is to use a Gaussian random process for the initial velocity $u(x, 0)$. Such fields have Fourier spectrum components with independent (uncorrelated) phases and a Gaussian distribution with variance

$$
\left\langle|\hat{u}(k, 0)|^{2}\right\rangle \propto k^{-1-2 h} .
$$

In the following, the statistical quantities at time $t$ are understood as ensemble averages over this Gaussian distribution and computed over many realizations. The results reported in this study are shown for $h=-1 / 2$ (white noise initial velocity), but they are insensitive to the choice of a precise negative value of $h$, corresponding to homogeneous initial velocity fields.

\subsection{Burgers turbulence}

We briefly describe here the numerical schemes and main phenomenological characteristics of the computed velocity field.

An interval of space of finite length $L_{\max }$ is discretized over $N$ grid points $(N=32768$ in the results reported here). Periodic boundary conditions are used to minimize end effects. One very nice feature of the Hopf-Cole solution (5) is that it can be discretized so that the solution at time $t$ can be computed from the initial conditions in a single step - thus avoiding integration over intermediates times. The computing cost of the maximization operation can be reduced from an apparent $N^{2}$ to $N \log _{2} N$ using the monotonicity of the inverse Lagrangian function as shown in [20] ( $N$ is the number of grid points). This computing efficiency allows the high resolution used here; it also permits to do a very large number of realizations to
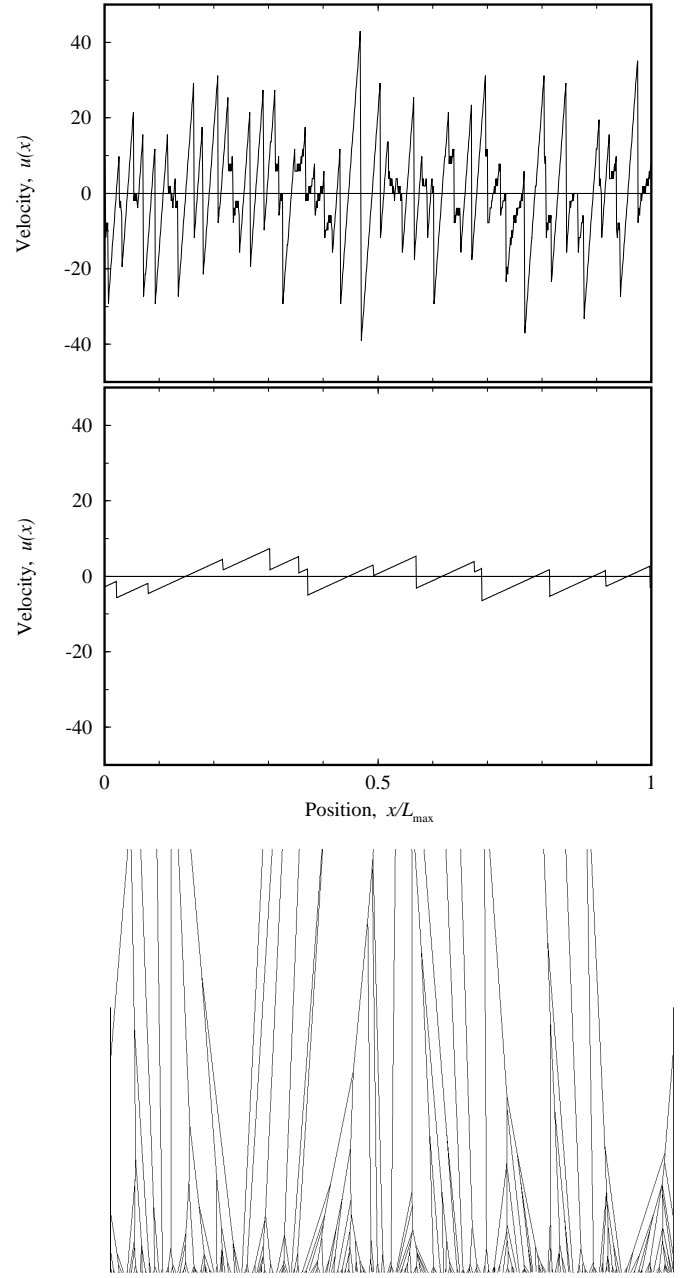

Fig. 1. Velocity profile for one realization of white noise initial velocity, shown at two successive times $t_{1}$ and $t_{2} \gg t_{1}$. Left: space-time diagram of the shock formation.

compute statistical properties (about $10^{7}$ realizations are used in the results reported here).

We show in figure 1 typical velocity profiles. The initial condition, as in all of the figures shown, is chosen with $h=$ $-1 / 2$, corresponding to an initial velocity field white in space. One observes that as times develops, the number of shocks decreases and their separation increases, due to the collision and merging of the shocks. In fact the mean separation between shocks varies with times as $t^{1 / 1-h}=$ $t^{2 / 3}$, like any length scale in the problem. The amplitude of the shocks decreases as $\ell(t) / t=t^{-1 / 3}$ because of the progressive cancellation of the velocities as shocks merge.

In this inelastic collision process, energy is dissipated. In a collision between two particles having the same mass $m$ and velocities $u_{1}$ and $u_{2}$, the energy loss is $m\left(u_{2}-\right.$ $\left.u_{1}\right)^{2} / 4$. The dissipation rate is thus proportional to $\left(u_{2}-\right.$ $\left.u_{1}\right)^{3} / \delta$ where $\delta$ is the initial separation of the particles. Note that dissipation is present in this system, even in the limit of vanishing viscosities. 


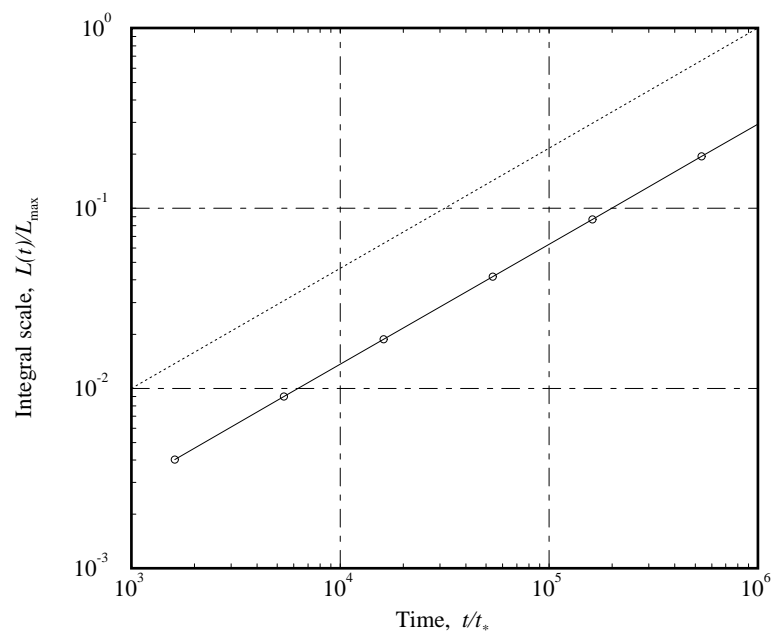

Fig. 2. Time evolution of the integral scale $L(t)$ for decaying Burgers turbulence with initial scaling exponent $h=-1 / 2$. The dotted line is the scaling prediction $L(t) \propto t^{2 / 3}$. The unit of time $t_{*} \equiv\left\langle\left[\partial_{x} u(x, 0)\right]^{2}\right\rangle^{-1 / 2}$ is set by the inverse of the standard deviation of the initial velocity gradient and corresponds to the time of the first shocks formations.

\subsection{Global variables in Burgers}

The preceding discussion was mostly phenomenological. We now define precisely the global quantities considered in this study. One very important parameter is the effective integral scale at time $t$. Since the turbulence is decaying, an integral length scale is a function of time, different from $L_{\max }$, the size of the physical domain. We define the integral length scale as

$$
L(t)=\left(\frac{\int_{0}^{\infty} \mathrm{d} k E(k, t) k^{-2}}{\int_{0}^{\infty} \mathrm{d} k E(k, t)}\right)^{1 / 2}=\left(\frac{\left\langle\psi^{2}(x, t)\right\rangle}{\left\langle u^{2}(x, t)\right\rangle}\right)^{1 / 2}
$$

where $E(k, t)$ is the velocity power spectral density in space at time $t ;\langle\bullet\rangle$ denotes ensemble averaging over the initial conditions, so that the last expression is valid at all positions by homogeneity. Other characteristic length scales could be defined on this system, a mean distance between shocks or a Taylor microscale for example; in fact they would all be proportional to one another since all length scales have the same evolution in time (equation (8)). Note that the characteristic length scale of the velocity auto-correlation function is also proportional to $L(t)$ and thus an increasing function of time.

Figure 2 shows that the integral scale perfectly follows the scaling argument (8). This is not surprising if the initial conditions and the equation are really scale-invariant, and should rather be taken as a check on the quality of the numerical simulations. It is only for very late times when the integral scale $L(t)$ approaches the system size $L_{\max }$ that scale invariance begins to be broken.

Two global quantities related to the energy balance are studied here: the total kinetic energy $E(t)$ and the total dissipation $\epsilon(t)$. The energy is defined as

$$
\begin{aligned}
E(t) & =\frac{1}{2 L_{\max }} \int_{0}^{L_{\max }} \mathrm{d} x u^{2}(x, t)=\overline{u^{2}(x, t)} \\
& =\frac{1}{\pi} \int_{0}^{\infty} \mathrm{d} k E(k, t)
\end{aligned}
$$

Strictly speaking this is the velocity variance, rather than the kinetic energy since the density is omitted. $\bullet$ denotes averaging over space, so that $E(t)$ can change for every realization, but does not depend on position.

The total dissipation can be defined even in the limit of vanishing viscosity by using the equivalent of the KármánHowarth relationship for the Burgers equation

$$
\frac{\partial\left\langle\left(\Delta_{r} u\right)^{3}\right\rangle}{\partial r}=-12 \epsilon(t)+3 \nu \frac{\partial^{2}\left\langle\left(\Delta_{r} u\right)^{2}\right\rangle}{\partial^{2} r}
$$

which in the limit of infinite Reynolds numbers yields

$$
\begin{aligned}
\epsilon(t) & \equiv \epsilon_{D}(t)=-\frac{\mathrm{d} E(t)}{\mathrm{d} t} \\
& =\frac{-1}{12} \sum_{\text {shocks }} \frac{\left[u\left(x_{s}+\delta / 2, t\right)-u\left(x_{s}-\delta / 2, t\right)\right]^{3}}{\delta}
\end{aligned}
$$

where $\delta$ is the width of a shock. In our simulations, $\delta$ is equal to the resolution in space $L_{\max } / N$. The dissipation is concentrated in the shocks, the ramps in the velocity profiles do not contribute to $\epsilon(t)$. Note that while the energy is distributed between the shocks and the dissipation is localized on the shocks, both quantities (ramps and shocks) are completely correlated. We stress that the definition of the total energy in equation (11) is consistent with the Kármán-Howarth balance (12).

Both global quantities $E(t)$ and $\epsilon(t)$ are defined as the sum over space of local positive defined quantities, namely the square of the velocity and the local dissipation. The probability density of the local energy $u^{2}(x, t) / 2$ (independent of position by homogeneity) is controlled by that of the single point velocity. The latter is known to be quasiGaussian in Burgers turbulence [13], so that the local energy is expected to be distributed as a $\chi^{2}$ law with one degree of freedom. This is confirmed in figure $3(\mathrm{a})$. The global energy is the sum of $N$ such positive variables. If the contributions are uncorrelated (and i.i.d.), the distribution of $E(t)$ should follow a $\chi^{2}$ law with $N$ degrees of freedom. In the limit of very large $N$ 's, it reduces to Gaussian. The argument is more complicated for the dissipation. Locally, the dissipation is related to the cube of the velocity increments whose distribution is non-Gaussian with a stretched exponential tail — see figure 3(b). However, we expect that the global dissipation follows again a $\chi^{2}$ law in the limit of large number $N$ of uncorrelated contributions. We show in the next section that this argument is incorrect at large times : asymmetric, strongly non-Gaussian distributions are observed for the global quantities. We link this effect to the development of large scale correlations in the system. 



Fig. 3. PDF of the local energy (a) and dissipation (b) at large time $t / t_{*}=1.6 \times 10^{5}$. The local energy is defined as (half) the square of the local velocity $u^{2}(x, t) / 2$ while the local dissipation is minus one twelfth the cube of the local velocity increment over the mesh size $\delta$, that is $-[u(x+\delta, t)-u(x, t)]^{3} / 12 \delta$.

\section{Results}

\subsection{Probability distributions of global variables}

We show in figure 4 the evolution with time of the PDF of the global energy $p(E)$ and dissipation $p(\epsilon)$. Emphasis is here on the shape of the distributions: the quantities have been shifted by their mean value $\left(\mu_{E} \equiv\langle E\rangle\right.$ and $\left.\mu_{\epsilon} \equiv\langle\epsilon\rangle\right)$ and rescaled by their standard deviation $\left(\sigma_{E} \equiv\left(\left\langle\left[E-\mu_{E}\right]^{2}\right\rangle\right)^{1 / 2}\right.$ and $\left.\sigma_{\epsilon} \equiv\left(\left\langle\left[\epsilon-\mu_{\epsilon}\right]^{2}\right\rangle\right)^{1 / 2}\right)$, so that all distribution have zero mean and unit variance. We first observe that the distributions are clearly non-Gaussian, even though the global quantities are calculated over a very large number of points. They are strongly asymmetric and develop exponential tails for fluctuations above the mean, as time increases. Quite surprisingly, the shape of the PDF and its evolution with time is the same for the energy and the dissipation, even if the latter is always slightly more skewed if considered at the same time.

This behavior is in agreement with previous measurements of energy injection in confined turbulent flows [18, 22 ] and of the fluctuations of the order parameter at a critical point $[1,5]$. For comparison, the PDF of the power injection $\epsilon_{I}$ in the confined turbulence experiment is also shown in figure 4 with the sign of the variable reversed since injection and dissipation have opposite variations.
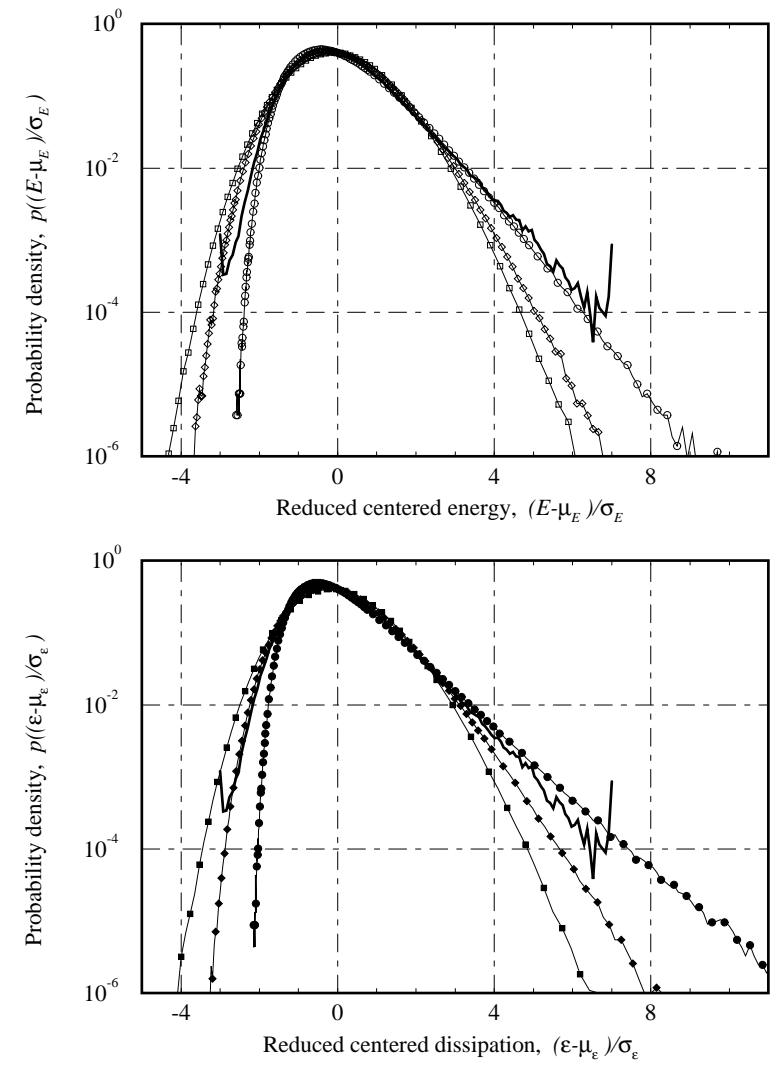

Fig. 4. PDFs $p\left(\left(E-\mu_{E}\right) / \sigma_{E}\right)$ of the global energy (a) and of global dissipation $p\left(\left(\epsilon-\mu_{\epsilon}\right) / \sigma_{\epsilon}\right)$ (b), shown at three successive times $t / t_{*}=1.6 \times 10^{3}(\square), 1.6 \times 10^{4}(\diamond), 1.6 \times 10^{5}(\circ)$. The thick line is the experimental PDF of $-\epsilon_{I}$ in the confined incompressible turbulence experiment.

Another feature is that $p\left(\left(E-\mu_{E}\right) / \sigma_{E}\right)$ and $p((\epsilon-$ $\left.\mu_{\epsilon}\right) / \sigma_{\epsilon}$ ) follow a continous transition with time, from a quasi-Gaussian form at small times to the non-Gaussian asymmetric shape at large times. This change is independent of the choice of the scaling exponent of the initial conditions which only sets the pace of the time evolution.

The evolution with time of the mean values and standard deviations of the global energy and of the global dissipation are shown in figure 5. All quantities decay in a self-similar way with time. The behavior of the mean values can be obtained from dimensional analysis. Consider for example the energy; dimensionally

$$
\mu_{E}(t) \sim\left(\frac{L(t)}{t}\right)^{2} \sim t^{2 h / 1-h},
$$

as verified in figure 5. For the dissipation one has

$$
\mu_{\epsilon}(t) \sim \frac{(L(t) / t)^{2}}{t} \sim t^{(3 h-1) /(1-h)} .
$$

We show in figure 5 that our numerical computations are in excellent agreement with the dimensional prediction. The standard deviations while also following an algebraic decay, have a different scaling exponent, which cannot be found by simple dimensional analysis. However 


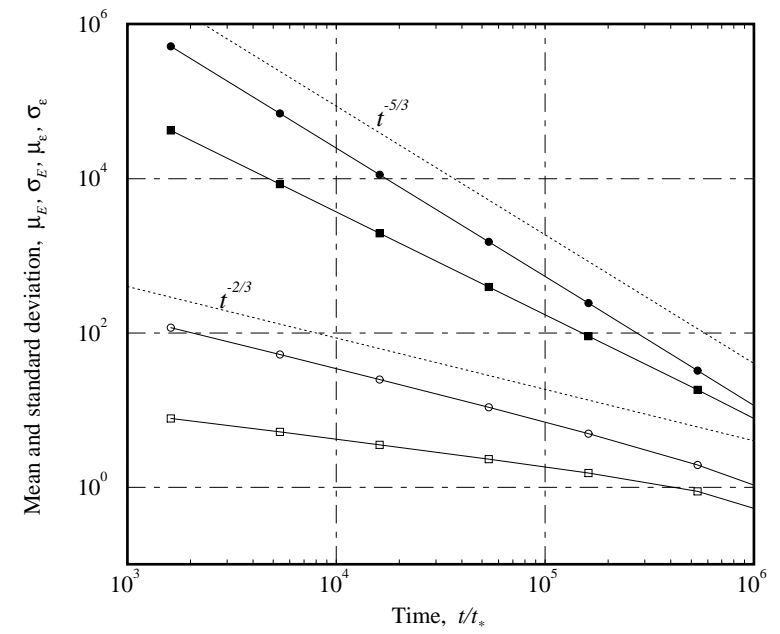

Fig. 5. Time evolution of the mean (o) and standard deviation $(\square)$ of the global energy (unfilled symbols) and global dissipation (filled symbols). For $h=-1 / 2$, the dimensional prediction for the scaling of the mean energy is $t^{-2 / 3}$ and the scaling of the mean dissipation is $t^{-5 / 3}$ (both shown as dotted lines).

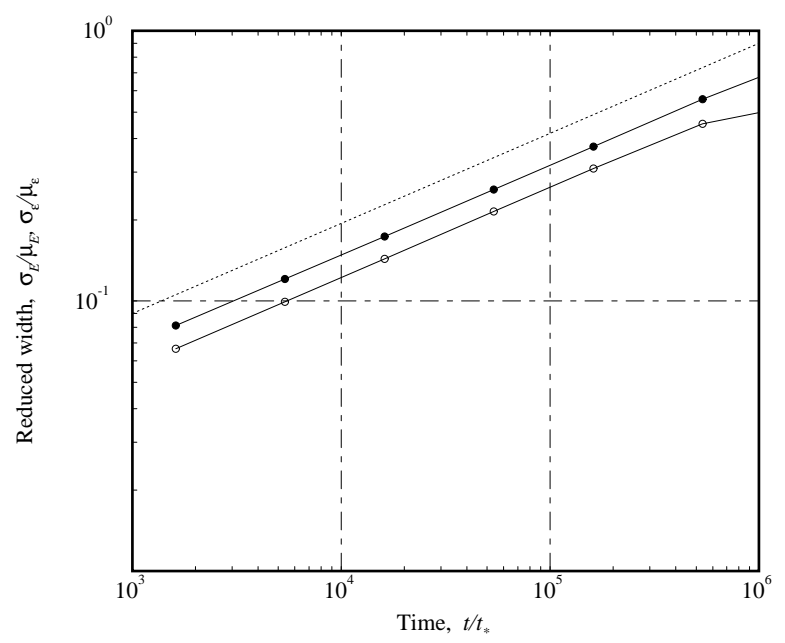

Fig. 6. Time evolution of the ratio $\sigma(t) / \mu(t)$ for the energy (unfilled symbols) and the dissipation (filled symbols). The dotted line corresponds to a power law $\propto t^{1 / 3} \sim L^{1 / 2}(t)$.

they can be compared to the mean values; a remarkable feature displayed in figure 6 is that we observe for both the energy and the dissipation

$$
\frac{\sigma(t)}{\mu(t)} \sim t^{\frac{1}{2} \frac{1}{1-h}} \sim L^{1 / 2}(t)
$$

In the following, we will show that this ratio can be interpreted as the inverse square root of the effective number of degrees of freedom in the system.

\subsection{Role of correlation length}

The above observations suggest that the only effective control parameter in this problem is the integral length scale $L(t)$. It plays the role of a correlation length for the velocity and thus for the local energy and the local dissipation. In this respect it was proposed in [4] that the existence of a macroscopic correlation length plays a fundamental role in the development of non-trivial fluctuations of the global quantity. In the case of a critical system, the 2DXY model, it has been shown in [23] that the ratio of the system size to the correlation length controls the shape of the global distribution. For small correlation length the PDF is almost Gaussian and goes to a limiting shape with an asymmetric exponential tail when the correlation length is comparable to the system size.

In our case, the velocity profile at large times consists of ramps extending over typical length $L(t)$ and amplitude $L(t) / t$, so that the velocity is indeed completely correlated over a length $L(t)$. Hence, one may view a system of total size $L_{\max }$ as $L_{\max } / L(t)$ nearly independent subsystems of size $L(t)$. In this picture, the effective number of degrees of freedom at time $t$ for a global quantity is

$$
\mathcal{N}(t)=\frac{L_{\max }}{L(t)} .
$$

Note that in this decaying problem, the effective number of degrees of freedom decreases with time. Recast in this frame, it is expected that the ratio of the mean to the standard deviation should scale as

$$
\frac{\mu(t)}{\sigma(t)} \sim \mathcal{N}^{1 / 2}(t)=\left(\frac{L_{\max }}{L(t)}\right)^{1 / 2}
$$

which is indeed what we observe.

Recalling that we are integrating positive quantities in each subsystem, we note that the property $\mu / \sigma \propto \mathcal{N}^{1 / 2}$ should be satisfied for any well-behaved probability density function for the global quantity. This is exactly the case for the $\chi^{2}$ distribution which is expected to appear in this problem. However, we show in figure 7 that it is not the right distribution. The $\chi^{2}$ distribution made with the observed effective number of degrees of freedom $\mathcal{N}=$ $2(\mu / \sigma)^{2}$ has indeed the correct width and mean value, and is closer than a Gaussian to the observed PDF even with a one-parameter fit, but does not correctly reproduce the exponential tail at large times. Note that in order to check this property the PDFs shown in figure 7 corresponds to quantities that are rescaled by their standard deviation but not shifted to zero mean. The agreement for the first two moments confirms that for the global energy and global dissipation, $\mathcal{N}$ does behave as an effective number of degrees of freedom, in a statistical sense. However, the distribution differ for higher-order moments. At large times the experimental PDFs deviate from the $\chi^{2}$ distribution by developing a pure exponential tail for values above the mean.

\subsection{Link with extremal statistics}

We now discuss the functional form of the PDF of the global quantities. The $\chi^{2}$ distribution or its equivalent 

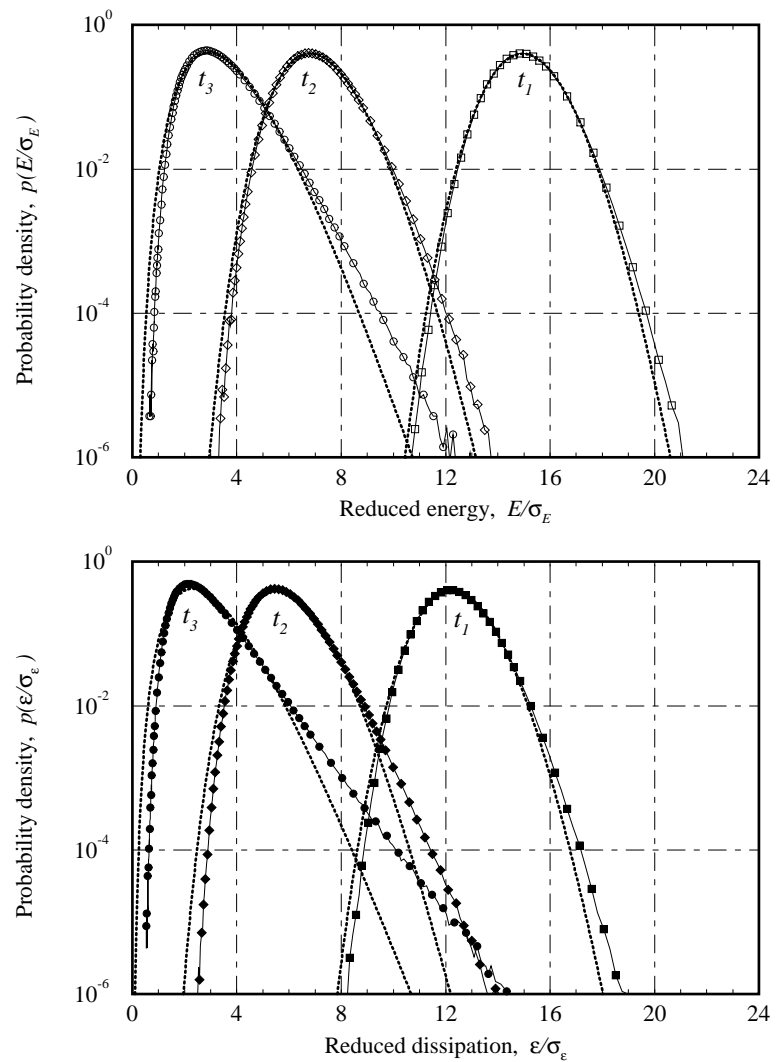

Fig. 7. Comparison of the PDFs $p\left(E / \sigma_{E}\right)\left(\right.$ a) and $p\left(\epsilon / \sigma_{\epsilon}\right)$ (b) at times $t_{1} / t_{*}=1.6 \times 10^{3}(\square), t_{2} / t_{*}=1.6 \times 10^{4}(\diamond), t_{3} / t_{*}=$ $1.6 \times 10^{5}(\mathrm{o})$ as in figure 4 compared to $\chi^{2}$ distributions having the same number of degrees of freedom $2(\mu / \sigma)^{2}$ (thick dotted lines).

$\Gamma$ form do not reproduce the behavior of the large deviations - see again figure 7 . One possible candidate is the generalized Gumbel family of distributions. These distributions share several properties with the experimental distributions : (i) they are asymmetric with a very fast decay for values below the mean and an exponential tail for values above the mean; (ii) they have been introduced in the context of extreme values statistics [12] and we expect them to play a role in the dynamics of the global variables. As time develops, the number of effective independent quasi-particles in the system becomes small. As the global quantities depend non-linearly on their velocities, we expect that only the largest shocks will contribute to the global energy or dissipation.

The definitions of the generalized Gumbel distributions are recalled in appendix. In the case of a reduced variable (zero mean, unit variance) $y \equiv\left(z-\mu_{z}\right) / \sigma_{z}$, the $\operatorname{Gumbel}(a)$ functional form is

$$
G_{a}(y)=W_{a} \exp \left\{-a\left[\beta_{a}\left(y-\alpha_{a}\right)+e^{-\beta_{a}\left(y-\alpha_{a}\right)}\right]\right\}
$$

where $a$ is the parameter defining the distribution and $W_{a}, \alpha_{a}, \beta_{a}$ ensure the normalization, zero mean and unit variance conditions.

The PDFs of the global energy and dissipation are shown in figure 8 together with the corresponding Gum-
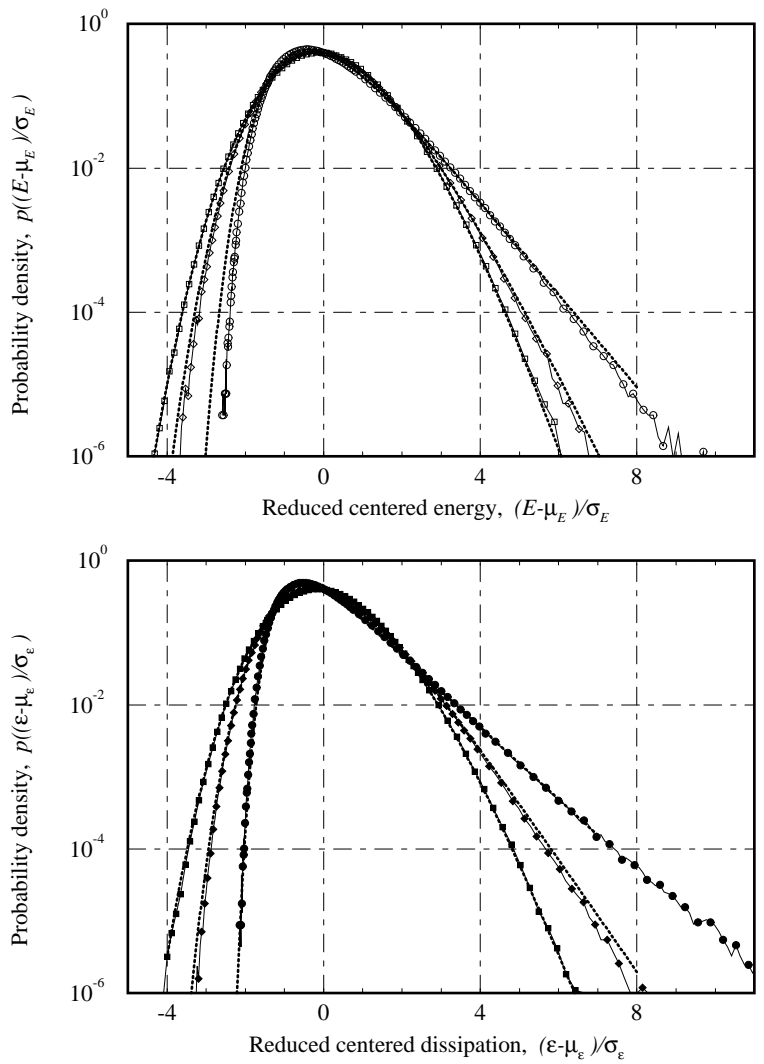

Fig. 8. Comparison of the PDFs of the global variables at times $t / t_{*}=1.6 \times 10^{3}(\square), 1.6 \times 10^{4}(\diamond), 1.6 \times 10^{5}(\circ)$ with a generalized Gumbel functional form (shown as thick dotted lines). (a) Global energy $p\left(\left(E-\mu_{E}\right) / \sigma_{E}\right)$ and corresponding Gumbel fits with parameters $a=20,6.3,1.6$. (b) Same for the dissipation $p\left(\left(\epsilon-\mu_{\epsilon}\right) / \sigma_{\epsilon}\right)$, the parameters of the best Gumbel fit are $a=12,2.7,0.57$.

bel best fits. We observe that the agreement is excellent, particularly for values above the mean. For values below the mean, small but systematic discrepancies exist at all times. We observe that the values of the parameter $a$ are not the same for the two global quantities; they are always larger for the energy distribution. The time evolution of $a(t)$ for both quantities is reported in figure 9 .

We observe that $a(t)$ varies in the same way for the global energy and for the dissipation and as the inverse of the integral scale, thus as the effective number of degrees of freedom $\mathcal{N}(t)$. In this sense, the parameter $a$ of the generalized Gumbel distribution plays the same role as the number of degrees of freedom in the $\chi^{2}$ distribution. This is a non trivial feature of the generalized Gumbel distribution family where the parameter $a$ is usually interpreted as the rank of the considered extreme event. However, it is possible to show that in the limit of large $a$ 's, the parameter $\beta_{a}$, which in the definition of the Gumbel plays the role of an inverse standard deviation, behaves as $\beta_{a} \rightarrow a^{-1 / 2}$. This property is in agreement the interpretation of $a$ as a number of degrees of freedom. 


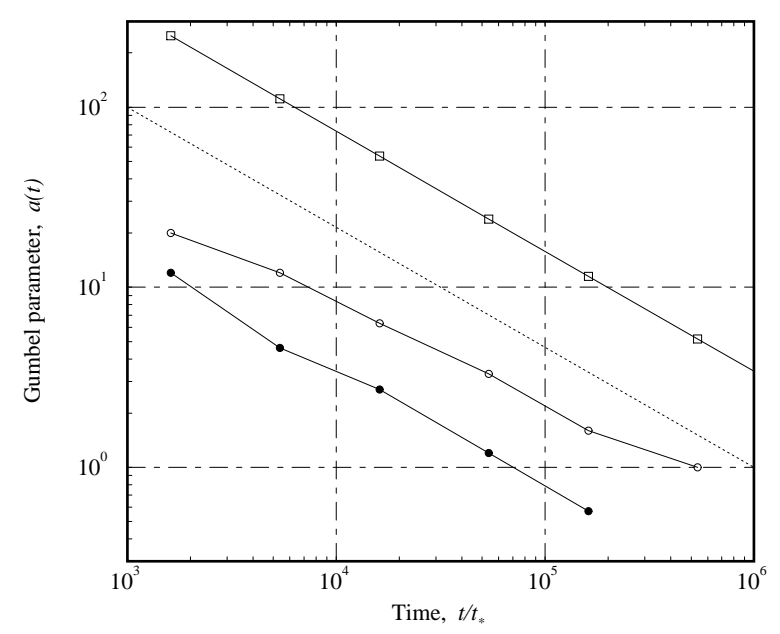

Fig. 9. Evolution with time of the best Gumbel fit parameter $a(t)$ for the energy PDF (unfilled $\circ$ ) and the dissipation (filled o) compared to that of the effective number of degrees of freedom $\mathcal{N}(t)=L_{\max } / L(t)(\square)$ and a power law $\propto t^{-2 / 3}$ (dotted line).

\subsection{Origin of exponential tails}

We now discuss in more details the role played by the statistics of large events and its link to the development of exponential tails in the global distribution at large times. Originally, the Gumbel ditribution with parameter $a$ describes the probability density function of the ath largest variable in a set of independent, identically distributed variables. It is thus interesting to study the distribution of extreme local values in our case. In the simulations we determine for every realization, the $m=1,2,3$ largest energy or dissipation events at each time. We then compute their individual probability distribution and the probability distribution of their cumulative sum.

Let us first consider the energy. We show in figure 10(a) the probability distribution for the three first largest local energies. The three curves collapse onto the same shape, that of the Gumbel(1) distribution function. This shows that the local largest events are not independent (otherwise the second and third largest should follow respectively Gumbel(2) and Gumbel(3) statistics). The statistics of the sum of the largest events is shown in figure 10(b). We observe that both the mean and the standard deviation increase linearly with the number of cumulated events. The curves are thus homothetic; this is a signature of completely correlated variables. Physically this is because all of these large events belong to the same velocity ramp in the limit of large times. The distribution of the sum of the local energies inside a given ramp is thus the same as that of the largest event in the ramp. The total energy in ramp results from the sum of $L(t) / \delta$ completely correlated variables, so that its mean is proportional to $L(t)$ and its variance to $L^{2}(t)$. Now, at any time $t$, there are $L_{\max } / L(t)$ such ramps so that:

- the average scales as

$$
\langle E\rangle=\frac{1}{N} \frac{L_{\max }}{L} \frac{L}{\delta}\left\langle u^{2}\right\rangle=\left\langle u^{2}\right\rangle,
$$
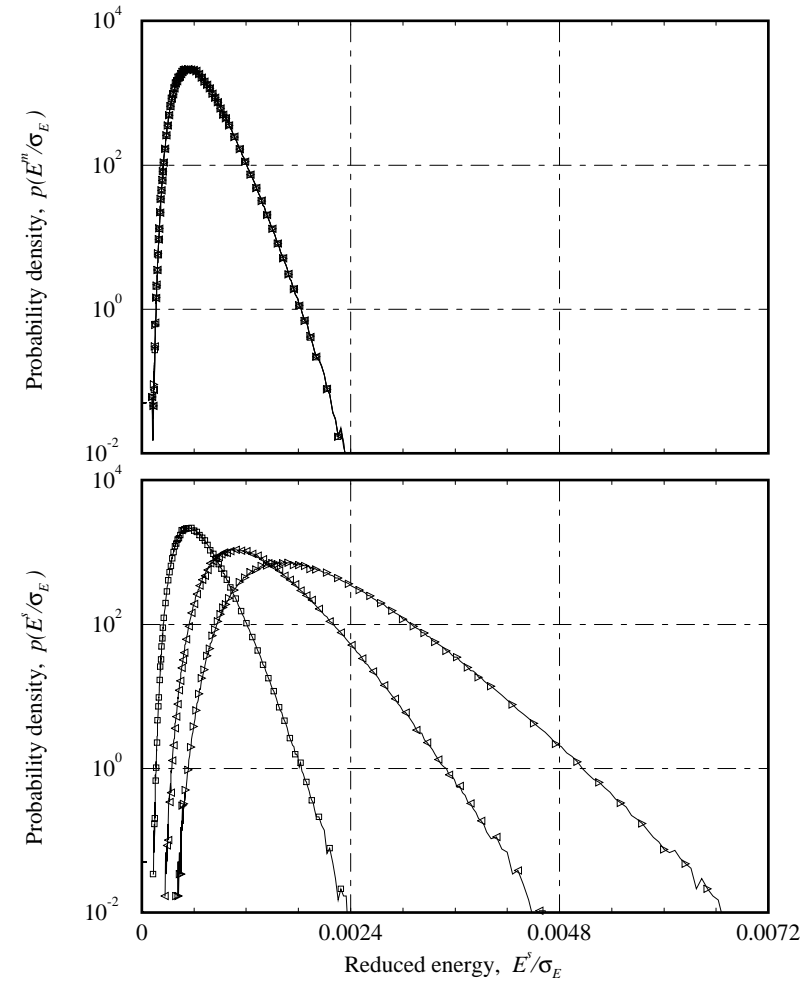

Fig. 10. Extreme values for the energy. (a) probability distribution of the first $(\square)$, second $(\triangleleft)$ and third $(\triangleright)$ largest local energy events, at a large time $t / t_{*}=1.6 \times 10^{5}$. The curves are not shifted by the mean and the normalization used here is that of the global standard deviation. All three distributions collapse on a single curve. (b) same for the distribution of the sum of these extreme events.

- the net variance scales as

$$
\left\langle E^{2}\right\rangle=\frac{1}{N^{2}} \frac{L_{\max }}{L}\left[\frac{L}{\delta}\left\langle u^{2}\right\rangle\right]^{2}=\frac{L}{L_{\max }}\langle E\rangle^{2},
$$

- the mean to standard deviation ratio behaves as

$$
\frac{\mu_{E}}{\sigma_{E}}=\left(\frac{L_{\max }}{L}\right)^{1 / 2}=\mathcal{N}^{1 / 2} .
$$

These predictions are in agreement with the experimental observations. In particular, they give the time dependence of the variance which could not be predicted by dimensional analysis. In addition, they imply that the ratio of the variance of the global energy to the variance of the local largest value varies as $\mathcal{N}^{-1}$, as we have verified from the numerical data. Finally, in this argument, one expects that the distribution for the total energy is given by the convolution ( $\mathcal{N}$ times) of homothetic Gumbel(1) functional forms. The exponential tail is thus brought here by the Gumbel(1) distribution. We observe that it is robust after a small number of convolutions.

We now consider the dissipation. At large times, it is concentrated in a small number of isolated and essentially uncorrelated shocks, so that both mean and variance are proportional to the number of shocks, which is 




Fig. 11. Extreme values for the dissipation. (a) probability distribution of the first $(\square)$, second $(\triangleleft)$ and third $(\triangleright)$ largest local dissipation events, compared to the distribution of the global dissipation (o), in the same conditions as in figure 10. (b) same for the distribution of the sum of these extreme events.

roughly $L_{\max } / L(t)$, that is $\mathcal{N}(t)$. The property

$$
\frac{\mu_{\epsilon}}{\sigma_{\epsilon}}=\left(\frac{L_{\max }}{L}\right)^{1 / 2}=\mathcal{N}^{1 / 2}
$$

is thus satisfied, but we stress again that the explanation is different for the energy and the dissipation. The global energy appears as the sum of $L^{-1}(t)$ uncorrelated terms, each involving $L(t)$ correlated variables, while the dissipation is the sum of $L^{-1}(t)$ uncorrelated variables. This different origin shows through in the fact that the ratio of the variance of the global dissipation to the variance of the largest dissipative event varies now as $\mathcal{N}$, the opposite as for the energy. The apparent number of degrees of freedom of the energy is always slightly larger than $\mathcal{N}$, because the velocity in the ramp is not completely correlated. Also, as can be observed in figure 11(a), the distribution for first, second and third largest local dissipation events are now correctly reproduced by the Gumbel(1), Gumbel(2) and Gumbel(3) functional forms. The distributions for the sums, shown in figure 11(b), tend rapidly to the limiting global distribution. The remarkable feature is that they all acquire the same exponential tail originating in the largest Gumbel(1) distribution. The explanation is that the higher rank distributions decay much faster the slope increase linearly with rank - so that at large deviations the asymptotic slope is set by the Gumbel(1) behavior. In the afore argument, the distribution considered pertain to variables reduced by the (constant) global standard deviation. To obtain the correct asymptotic slope for the global dissipation from the Gumbel(1), one must take into account the ratio $\sigma_{\epsilon} / \sigma_{\epsilon 1}$ of the variance of the two distributions. Hence, the parameter $a$ for the global distribution is such that the $G_{a}$ function has an exponential decay with a slope equal to that of the Gumbel(1) multiplied by $\sigma_{\epsilon} / \sigma_{\epsilon 1}$. Once again, the exponential tail in the global distribution is borne out of the Gumbel(1) distribution of the largest local events.

\section{Concluding remarks}

The Burgers equation proves to be a very fruitful tool to study the dynamics of global quantities. In the decaying regime, it has an integral scale $L(t)$ which varies continuously with time, so that the ratio of the system size to the effective correlation length can be tuned at will. Numerically one finds that the distributions of the global energy and dissipation change continuously. They have a near Gaussian shape at small times and develop an asymmetric exponential tail with time. We have found that the parameter that controls this transition is the effective number of degrees of freedom $\mathcal{N}(t)=L_{\max } / L(t)$. At all times the distribution have the property that $\mu / \sigma \propto \mathcal{N}^{1 / 2}$. This confirms that the global energy and dissipation are the right objects for the statistical decription of this system. The functional form of the distributions is very closely reproduced by the generalized Gumbel distributions, for which two interesting properties are observed: (i) their control parameter can be viewed as a number of degrees of freedom and (ii) they are quasi-stable with respect to convolution. Finally, in this system, the exponential tail for large deviations above the mean can be attributed to the statistics of the largest local events, i.e. the Gumbel(1) distribution.

This exceptional stability of the generalized Gumbel distributions deserves further investigations, in particular its link to a 'generalized' central limit theorem. Can the distribution of the sum of Gumbel distributed variables be represented by another Gumbel distribution ? By this, we mean the same semi-stable property as the $\chi^{2}$ (or $\Gamma$ ) distributions in which the number of degrees of freedom simply add up. In that case what is the composition rule for the control parameters $a$ ? We don't think the Gumbel distributions are exactly semi-stable, as the global distributions show some small but systematic deviations from the Gumbel form for values below the mean, but the stability of the exponential tail deserves to be examinated.

The physical origin of the robustness and apparent universality of the global PDFs is also intriguing. In confined decaying Burgers turbulence, the integral scale $L(t)$ goes through all values until it reaches approximately the size of the system, and it is only in that case that the global distributions reproduce the experimental ones or those of equilibrium statistical systems. In the latter case, it is clear that the correlation length is also of the same order as the size of the system, but the way a precise value is enforced is not at all clear. This is even less understood for the forced 
turbulence experiment, for which the energy injection is done at large scales, but the system has to create structures ranging from the dissipative scales (so that energy can be removed) up to the integral scales (if the number of degrees of freedom is really small). Also, the structures in Burgers are dissipative ones, and the energy appears completely slaved and correlated between these. Even though some type of localized dissipative structures have been observed in incompressible Navier-Stokes turbulence [3,21], the behavior of the velocity field between them is as yet unknown.

Another open question is to find the necessary ingredients for the distribution of the local variables, so that the global quantity converges to a Gumbel-type distribution. All local objects considered here are positive definite with an asymmetric PDF showing an exponential or stretched exponential tail. It is not yet clear whether these properties are sufficient and, if not, which would be needed in a more general context.

\section{Acknowledgements}

This work was motivated by discussions during the CECAM workshop on the statistics of global fluctuations, Lyon, March 2000. It was supported by contracts ACI No. 2226 from the French Ministère de la Recherche, the European Network Non-ideal Turbulence No. HPRN-CT2000-00162 and the BQR program of Observatoire de la Côte d'Azur. It is a pleasure to thank Mickael Bourgoin, Marc-Etienne Brachet, Sergey Gurbatov, Peter Holdsworth and Baptiste Portelli for fruitful discussions.

\section{Appendix : Gumbel distributions}

In a realization of $n$ independent measures $\left\{z_{i}\right\}_{i=1, \ldots, n}$ of a random variable with probability density function $f(z)$, the distribution for the $a$ th largest value $z_{a}$ tends for large values of $n$ to a limiting form found by Gumbel [12] that becomes independent of $f(z)$ and is

$$
G_{a}(z)=\frac{a^{a} \theta_{a}}{\Gamma(a)} \exp \left\{-a\left[\theta_{a}\left(z_{a}-u_{a}\right)+e^{-\theta_{a}\left(z_{a}-u_{a}\right)}\right]\right\} .
$$

Here, $u_{a}$ is the value of $z$ such that there are typically $a$ values among the $n$ ones which are greater than $u_{a}$, i.e. $F\left(u_{a}\right) \equiv \int_{-\infty}^{u_{a}} \mathrm{~d} z f(z)=1-a / n$. The intensity $\theta_{a}$ is defined as $\theta_{a}=(n / a) f\left(u_{a}\right)$.

The mean value $\mu_{a}$ and standard deviation $\sigma_{a}$ of the distribution of $z_{a}$ are related to the constitutive parameters $u_{a}$ and $\theta_{a}$ through

$$
\begin{gathered}
\mu_{a}-u_{a}=\frac{1}{\Gamma} \frac{\mathrm{d} \Gamma}{\mathrm{d} a}-\ln a \\
\left(\sigma_{a} \theta_{a}\right)^{2}=\frac{1}{\Gamma} \frac{\mathrm{d}^{2} \Gamma}{\mathrm{d} a^{2}}-\left(\frac{1}{\Gamma} \frac{\mathrm{d} \Gamma}{\mathrm{d} a}\right)^{2} .
\end{gathered}
$$

When the variable is considered in reduced units

$$
y \equiv \frac{z_{a}-\mu_{a}}{\sigma_{a}}
$$

so that it has zero mean and unit variance, its Gumbel $(a)$ distribution is given by

$$
G_{a}(y)=W_{a} \exp \left\{-a\left[\beta_{a}\left(y-\alpha_{a}\right)+e^{-\beta_{a}\left(y-\alpha_{a}\right)}\right]\right\}
$$

with

$$
\begin{gathered}
\beta_{a}=\left[\frac{1}{\Gamma} \frac{\mathrm{d}^{2} \Gamma}{\mathrm{d} a^{2}}-\left(\frac{1}{\Gamma} \frac{\mathrm{d} \Gamma}{\mathrm{d} a}\right)^{2}\right]^{1 / 2}, \\
\alpha_{a}=\frac{1}{\beta_{a}}\left(\ln a-\frac{1}{\Gamma} \frac{\mathrm{d} \Gamma}{\mathrm{d} a}\right)
\end{gathered}
$$

and

$$
W_{a}=\frac{\beta_{a} a^{a}}{\Gamma(a)}
$$

\section{References}

1. P. Archambault, S. Bramwell, J.-Y. Fortin, P.C.W. Holdsworth, S. Peysson and J.-F. Pinton, Universal magnetic fluctuations in the 2DXY model, Journal of Applied Physics, 83, 1-8, (1998).

2. S. Aumaître and S. Fauve, Journal de Chimie-Physique, 96, 1038-1043, (1999).

3. F. Belin, J. Maurer, P. Tabeling and H. Willaime, Observation of intense filaments in fully developed turbulence, J. Phys. II, 6, 573-584, (1996).

4. S.T. Bramwell, P.C.W. Holdsworth and J.-F. Pinton, Universality of rare fluctuations in turbulence and critical phenomena, Nature, 396, 552-554, (1998).

5. S.T. Bramwell, K. Christensen, J.-Y. Fortin, P.C.W. Holdsworth, H.J. Jensen, S. Lise, J. López, M. Nicodemi, J.-F. Pinton and M. Sellitto, Universal Fluctuations in Correlated Systems, Phys. Rev. Lett., 84, 3744, (2000).

6. S.T. Bramwell, J.-Y. Fortin, P.C.W. Holdsworth, S. Peysson, J.-B. Portelli and M. Sellitto, Magnetic fluctuations in the classical XY model: the origin of an exponential tail in a complex system, Phys. Rev. E, 63(4), (2001).

7. J.M. Burgers, On the application of statistical mechanics to the theory of turbulent fluid motion, Proc. Roy. Neth. Acad. Soc., 32, 643, (1929).

8. J.M. Burgers, The Nonlinear Diffusion Equation, D. Reidel Publ. Co., (1974).

9. J.D. Cole, On a quasi-linear parabolic equation occuring in aerodynamics, Quart. Appl. Math., 9, 225-236, (1951).

10. J. Farago, Injected power fluctuations in Langevin equation, submitted to J. Stat. Phys., arXiv:condmat/0106191, (2001).

11. J.D. Fournier and U. Frisch, L'équation de Burgers déterministe et statistique, J. de Méc. Théor. et Appl., 2, 699-750, (1983).

12. E.J. Gumbel, Statistics of Extremes, Columbia University Press, (1958). 
13. S. Gurbatov, A. Malakhov and A. Saichev, Nonlinear random waves and turbulence in nondispersive media: waves, rays, particles, Manchester University Press, (1991).

14. E. Hopf, The partial differential equation $u_{t}+u u_{x}=u_{x x}$, Comm. Pure Appl. Mech., 3, 201-230, (1950).

15. M. Kardar, G. Parisi and Y.C. Zhang, Dynamical scaling of growing interfaces, Phys. Rev. Lett., 56, 889-892, (1986).

16. S. Kida, Asymptotic properties of Burgers turbulence, J. Fluid Mech., 93, 337-377, (1979).

17. Y. Kuramoto, Chemical Oscillations, Waves and Turbulence, Springer-Verlag, Berlin, (1985).

18. R. Labbé, J.-F. Pinton and S. Fauve, Power fluctuations in turbulent swirling flows, J. Phys. II, 6, 1-19, (1996).

19. S. Aumaître, S. Fauve, S. MacNamara and P. Poggi, Power injected in dissipative systems and the fluctuation theorem, Eur. Phys. J., B19, 449-460, (2001).

20. A. Noullez and M. Vergassola, A fast algorithm for discrete Legendre transforms, J. Sci. Comp., 9, 259-281, (1994).

21. A. Noullez, G. Wallace, W. Lempert, R.B. Miles and U. Frisch, J. Fluid Mech., 339, 287-307, (1997).

22. J.-F. Pinton, R. Labbé and P.C.W. Holdsworth, Power fluctuations in a closed turbulent shear flow, Phys. Rev. E, 60, R2452-R2455, (1999).

23. B. Portelli, P. Holdsworth, M. Selitto, and S. Bramwell, Universal magnetic fluctuations with a field induced length scale, Phys. Rev. E., 64, 036111, (2001).

24. A. Pumir, Phys. Fluids A, 8, 3112 (1996).

25. M. Schwartz and S.F. Edwards, Nonlinear deposition: a new approach, Europhys. Lett., 20, 301-305, (1992).

26. S.F. Shandarin and Ya.B. Zeldovich, The large-scale structure of the universe: Turbulence, intermittency, structures in a self-gravitating medium, Rev. Mod. Phys., 61, 185220, (1989).

27. Z.S. She, E. Aurell and U. Frisch, The inviscid Burgers equation with initial data of brownian type, Comm. Math. Phys., 148, 623-641, (1992).

28. M. Vergassola, B. Dubrulle, U. Frisch and A. Noullez, Burgers' equation, Devil's staircases and the mass distribution for large-scale structures, Astron. Ef Astrophys., 289, 325-356, (1994). 